Facultad de Psicología

Carrera de Psicología

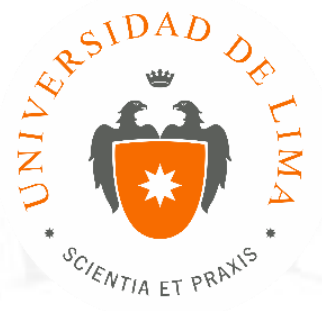

\title{
ACOMPAÑAMIENTO EN EL PROCESO DE ADAPTACIÓN ESCOLAR A NIÑOS DE 2 A 5 AÑOS DE UNA INSTITUCIÓN EDUCATIVA PRIVADA
}

Trabajo de suficiencia profesional para optar el título profesional de Licenciado en Psicología

\section{Ana Paula Baldovino Barreto}

Código 20102197

$$
\text { Lima - Perú }
$$

Febrero de 2019 


\section{ACOMPAÑAMIENTO EN EL PROCESO DE ADAPTACIÓN ESCOLAR A NIÑOS DE 2 A 5 AÑOS DE UNA INSTITUCIÓN EDUCATIVA PRIVADA}




\section{TABLA DE CONTENIDO}

INTRODUCCIÓN 6

CAPÍTULO 1: IDENTIFICACIÓN DEL PROBLEMA ........................................................7

CAPÍTULO 2: DESCRIPCIÓN DE LAS ACTIVIDADES REALIZADAS ..................... 11

2.1 Acompañamiento a los niños de 2 a 5 años en el proceso de adaptación escolar ....... 12

2.2 Capacitación a las cuidadoras sobre el rol del acompañante en el proceso de adaptación escolar .

2.2.1 Taller: Capacitación para cuidadoras y acompañantes en la adaptación del

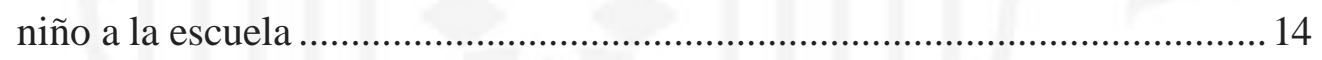

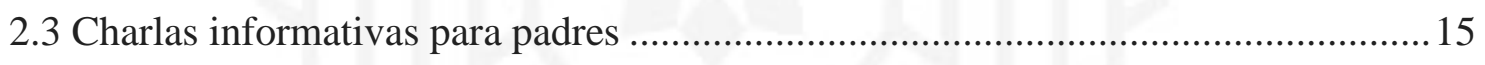

2.3.1 Taller: Generando un vínculo saludable con mi hijo ................................... 15

CAPÍTULO 3: RESULTADOS DELA INTERVENCIÓN ..................................................17

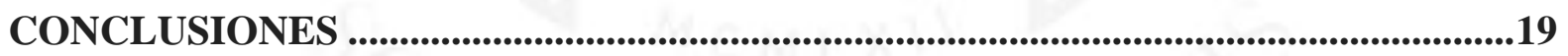

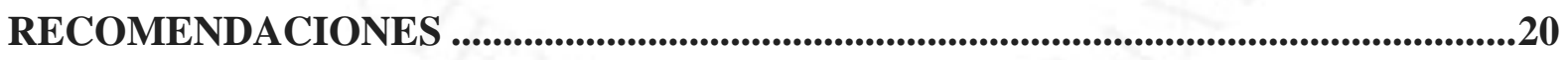

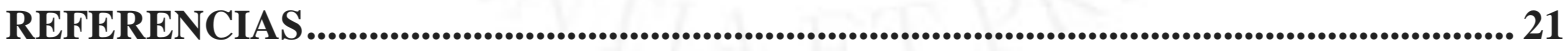

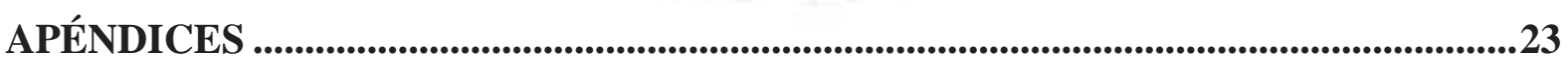




\section{ÍNDICE DE APENDICES}

Apéndice 1: Modelo de entrevista con padres......................................................... 24

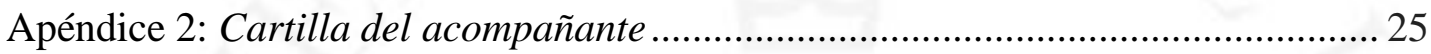

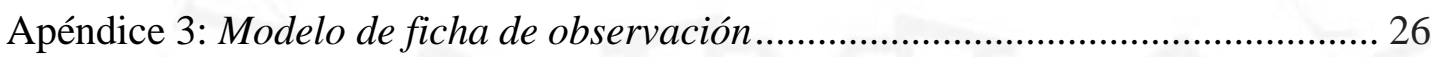

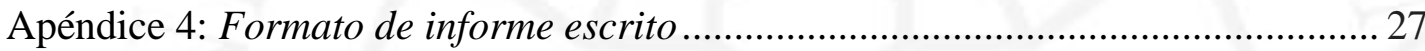

Apéndice 5: Formato de inscripción para capacitación de cuidadoras ......................28

Apéndice 6: Formato de inscripción de taller para padres........................................29 


\section{INTRODUCCIÓN}

En la actualidad, la educación inicial es muy importante debido a que permite el desarrollo y la formación del niño en colaboración con la familia. Dentro de esta situación, en los nuevos contextos se están recibiendo a niños desde muy temprana edad, es así que la escuela en muchos casos es un hogar temporal, porque los padres ya sea por motivos laborales, o, por otros de tipo social, no pueden estar con sus hijos en esta etapa tan crucial para su desarrollo.

Esta situación es la que determina la aparición de organizaciones educativas especializadas para encargarse del desarrollo emocional y cognitivo de los niños tempranamente. Londoño (2013) analiza cuatro componentes necesarios para el apoyo requerido por los niños que inician la etapa escolar: considerar las características del desarrollo de los niños, reconocer los factores que influyen en la adaptación a nuevas situaciones, establecer relaciones con las familias, implementar estrategias que ayuden a los niños durante la transición. Es así que la escuela, toma un valor fundamental al ser el nuevo espacio de contención para el niño, es por ello que es importante trabajar el tema de la adaptación analizando los niveles de apego con los que cuenta el niño y las condiciones en las que se encuentra para poder ayudarlo en este proceso, reforzando al espacio como uno amigable, seguro y acogedor que pueda asemejarse a su hogar. Según Geddes (2010), una escuela se muestra como una base segura cuando el alumnado puede funcionar con eficacia emocional y cognitiva.

De alguna manera, el departamento psicológico tiene una intervención fundamental para colaborar con el proceso de los niños en la adaptación a la institución. Generando oportunidades de desarrollo óptimo basándose en los principios de la filosofía educativa Reggio Emilia que se sostiene en la educación socio constructivista con la que trabaja la institución. 


\section{CAPÍTULO I IDENTIFICACIÓN DEL PROBLEMA}

Durante la primera infancia, los bebés crean un vínculo afectivo que se desarrolla con la persona que lo atiende y cuida, este vínculo es denominado apego. Según Moneta (2014), la teoría del apego desarrollada por Bowlby durante los años 1969 a 1980, describe el efecto que producen las experiencias tempranas y la relación de la primera figura vincular en el desarrollo del niño. Asimismo, menciona que el apego hacia personas significativas, ya sean estos progenitores, maestros o cuidadoras acompaña al niño durante el desarrollo de la primera infancia y es la base para tener mayores posibilidades de tener experiencias emocionales positivas durante la primera etapa de vida.

Según Geenen (2014) existen cuatro fases en las cuales se desarrolla el apego: la primera fase inicia de 0-3 meses donde el bebé envía señales, la segunda fase de 4-6 meses donde el bebé reacciona positivamente a determinadas personas de su entorno, la tercera fase de 6-8 meses bebé muestra señales y movimientos, prefiere a una persona específica; la ansiedad de separación surge cuando la persona cuidadora se retira y a partir de los 12 meses se puede determinar la calidad del apego en relación con cada persona. Por último, la cuarta fase que se presenta a partir de los dos años en donde el niño se pone en el lugar de la persona con quien ha desarrollado ese vínculo.

Geddes (2010) menciona que el apego seguro es un factor que se relaciona con el éxito del niño en la escuela, involucrando lo social, curiosidad, juego, exploración, buenas relaciones con sus pares y aceptación de límites impuestos por el docente. Es decir, la persona a cargo de la adaptación debe brindarle seguridad y autonomía, así como fomentar la sensibilidad a las necesidades del niño tomando en cuenta la afectividad, la apertura hacia el niño, la variedad de juegos, el sentido del momento preciso y el tiempo del niño para adaptarse a nuevos espacios sin apresurarlo, lo cual permitirá una adaptación optima a nuevos vínculos. 
Luego de la primera etapa de vínculo que se genera con los padres y/o cuidadora en los primeros dos años de vida. El niño inicia el periodo pre escolar, ingresando al nido en donde se presentan nuevos retos. Es dentro de este periodo donde se da el proceso de adaptación hacia un espacio diferente, empezando nuevos vínculos que se establecen entre los niños, padres y maestros para lograr que el niño pueda permanecer de manera autónoma e independiente en el espacio sin la necesidad de un acompañante permanente. (Bautista Loza \& Espinosa Anchaluisa, 2010)

Vélez Vasco (2015) menciona un aspecto importante sobre la relación familia-escuela, señalando que el hecho de que la madre cree una buena relación con la maestra le dará seguridad al niño y de esta manera ella podrá ir identificando las relaciones primarias con la familia del niño e incluso aportar a la crianza que se le ha dado hasta el momento. Tal como lo señala Woods Winnicott (1986, p.24) "la función del jardín de infantes, no consiste en sustituir a una madre ausente, sino en complementar y ampliar el papel que solo la madre puede desempeñar en los primeros años de la vida del niño".

En esta etapa pre escolar se relacionan tres contextos importantes: familiar, educativo y social. La relación entre la familia y la escuela se sitúa en un contexto histórico e institucional. Se ubica en la articulación de dos tipos de instituciones con poderes asimétricos, así como en un contexto social. La escuela es un lugar de encuentro y comunicación para los familiares de los niños y niñas que acuden a ese centro, por ello la integración entre ambas instituciones, requiere de una comunicación y la posibilidad de compartir elementos comunes. (Gómez Martínez, 2018)

Es así que estos tres contextos, influyen en el desarrollo integral del niño durante esta nueva etapa. El primero, le brinda la seguridad al niño para permanecer en un nuevo espacio de manera tranquila y cómoda. El segundo, le brinda las estrategias para iniciar una etapa de 
exploración y contención buscando nuevas figuras de vínculo y protección (maestras) y finalmente el tercero, le permite generar nuevas relaciones con sus pares para crear progresivamente un juego y habilidades sociales que le permitan interactuar de manera segura. Sainz de Vicuña (2009) menciona que existen diferentes modos de comunicación con las familias lo que permite construir una relación que se basa en la confianza mutua y el respeto. Debido a que los contextos de la familia y de la escuela comparten la socialización de los niños y las niñas desde edades cada vez más tempranas, se presenta una mayor participación de los padres que facilite la inclusión del ámbito familiar en la institución educativa para favorecer el desarrollo y el aprendizaje de los niños. Este proceso de adaptación se debe dar de manera progresiva, respetando los tiempos de cada niño, es aquí donde ellos observan, descubren y exploran el nuevo espacio reconociéndolo y formando vínculos seguros con las personas que lo rodean. Asimismo, este proceso involucra el tiempo que tiene cada niño para asumir la perdida y la ganancia que le supone la separación de su familia y/o acompañante dentro de esta etapa. Es importante, que la persona muestre serenidad y seguridad ayudando a que este periodo se desarrolle de la manera más dócil y amable posible. Sin embargo, el proceso de adaptación que se presenta durante esta etapa depende de la metodología educativa y el acompañamiento respectivo que se haga en cada caso en conjunto con los padres. (Geenen, Corveleyn, \& Koroleff, 2014)

Navia Antezana \& Villareal Delgado (2013); Diloné y Pons (2010); y Vélez Vasco (2015) definen acompañamiento desde una perspectiva relacional, desde este punto de vista se le da valor a la otra persona, se forman mutuamente permitiendo la subjetividad, el saber y las experiencias. Además, sostienen que el acompañamiento pedagógico es como una mediación de formación en centro y para la vida, desde donde se recrea la dinámica relaciona de la acción educativa y se aporta sentido vinculante a los nuevos conocimientos y competencias docentes. 
En la experiencia actual se han podido observar casos en los cuales los niños ingresan al nido y luego de un par de días acompañados se sienten seguros y confiados para lograr despedirse de manera tranquila y permanecer con la maestra sin necesidad del acompañante. Sin embargo, hay niños a los que les toma un tiempo más prolongado y esta adaptación debe realizarse de manera progresiva, el acompañante debe aprender ciertas pautas para que le brinde la seguridad y el espacio al niño para que se vincule con las maestras y pares; de manera que, permita la intervención de la maestra en ciertas actividades como la interacción en el juego o los momentos de aseo creando momentos de a dos que le permitan al niño sentirse acompañado y tranquilo sin la necesidad de acudir al cuidador principal.

Vélez Vasco \& Vergara M (2015) señalan que el acompañante debe trasladar la confianza hacia el nuevo adulto que se encuentra a cargo del niño en este nuevo espacio para que entienda que este podrá ayudarlo y acompañarlo si lo necesita. Este tipo de adaptación amable y secuencial que respeta el tiempo y momento en el que el niño se encuentra seguro, permite se formen de manera más segura y puedan poco a poco enfrentar diferentes retos propios de la edad con un acompañamiento que les permita buscar las estrategias para lograr lo que desean y creando soluciones diferentes, de acuerdo a los recursos con los que cada uno cuenta. Asimismo, minimiza la angustia o frustración que pueden sentir al ser separados de manera repentina sin entender el proceso y sintiéndose inseguros.

Como se ha mencionado en el párrafo anterior, el rol del docente es primordial ya que brinda las estrategias necesarias hacia los acompañantes y padres que inician esta nueva etapa con dudas e inseguridades. Los docentes deben brindar la confianza y estabilidad necesaria para que tanto los padres como los niños se sientan cómodos en este nuevo espacio. (Yábar Tito \& Bronzoni Zlatar, 2018)

La educación inicial ha presentado cambios durante estos últimos años, es así que se han incorporado nuevos métodos y filosofías de enseñanza en diferentes instituciones que buscan 
una alternativa educativa diferente a la tradicional, la cual se enfoca en mayor medida en las necesidades del niño, el acompañamiento del maestro y los padres dentro de este proceso de aprendizaje y adaptación al nuevo espacio. Esto ha permitido que el proceso de adaptación escolar se presente de manera más amable dentro de estas instituciones como es el caso de XYZ. Esta es una de las instituciones basadas en las teorías de aprendizaje socio constructivistas y de aprendizaje significativo, como es la filosofía Reggio Emilia. (Yábar Tito \& Bronzoni Zlatar, 2018)

La pedagogía de Reggio Emilia, nace en un pueblo llamado Reggio Emilia al norte de Italia, gracias a su fundador, el pedagogo y profesor Loris Malaguzzi. Hacia el año 1945 nace el primer nido. Más tarde en 1963, la comuna de Reggio Emilia comenzó a instituir su propia red de servicios educativos, con la creación de las primeras escuelas de infancia. Hoy en día, la filosofía de Reggio Emilia es reconocida mundialmente como una de las mejores propuestas educativas para la primera infancia. Esta filosofía se centra en cinco principios básicos: El niño como protagonista, docente competente, colaborador, investigador y guía, espacio como tercer maestro, las familias como aliadas y la documentación pedagógica (Valencia Chávez, 2017). Basado en estos principios y en la imagen que se tiene del niño desde esta filosofía educativa de escucha y comprensión, el departamento de psicología de la institución en conjunto con el equipo de docentes se encarga de que el acompañamiento de adaptación escolar se presente de manera amable, enfatizando la acogida y el respeto hacia los tiempos de cada alumno. Su filosofía entiende la participación de la familia como un aspecto necesario e indiscutible para conseguir seguridad emocional en los más pequeños, ello les ayuda en la construcción de su propia identidad personal y social. Según Valencia Chávez (2010), se puede hablar de educación siempre y cuando exista un proyecto compartido familia-escuela. A partir de esto, se realiza un trabajo personalizado con cada familia para el seguimiento y asesoramiento de este periodo brindándole las estrategias necesarias. 


\section{CAPÍTULO II: DESCRIPCIÓN DE LAS ACTIVIDADES Y TAREAS REALIZADAS}

\section{1 Acompañamiento de los niños de 2 a 5 años en el proceso de adaptación escolar}

El niño que entra desde temprana edad a la escuela experimenta nuevas sensaciones, la separación del ambiente de casa y de la familia. Esta experiencia de cambio, genera en muchos de ellos momentos de ansiedad, rabia y temor los cuales expresan de muchas formas: llanto, manifestaciones de rechazo a la escuela y a sus compañeros, estados fóbicos y en algunos casos agresividad. (Geddes, 2010) señala que la ansiedad surge como resultado de intentar mantener la proximidad adecuada y la rabia, como reproche de la ausencia de la figura de apego. De manera que, es una experiencia que requiere de cambios en su comportamiento, cambios para adaptarse al nuevo ambiente, a la escuela y a los nuevos vínculos que formará.

Para favorecer el proceso de adaptación del niño a la escuela, se inicia con una reunión hacia los padres de familia, guiada por la docente y la practicante de psicología, la finalidad de esta reunión es conocer aspectos importantes acerca del niño, si ha tenido alguna otra experiencia pre escolar, como son sus rutinas o tiempos libres y saber sobre su desarrollo a lo largo de sus primeros años de vida (Anexo 1). Asimismo, conocer quien lo acompañará en este proceso de adaptación e informar sobre ciertas pautas de conducta que deben tener en cuenta los que asistirán al proceso de adaptación del niño a la escuela, además de las características y de los roles de la acompañante dentro de la propuesta educativa que brinda XYZ (Anexo 2).

Luego se realiza una observación participante, en la que el observador interactúa con los sujetos este tipo de observación guarda relación con lo que plantea la institución sobre la interacción con los niños por medio del juego, tomando en cuenta un formato dado por la escuela. (Hernández Sampieri, Fernández Collado, \& Baptista Lucio, 2010),

La practicante de psicología tiene como objetivo observar y registrar diferentes momentos de la rutina del salón a cargo, en conjunto con ciertas conductas dependiendo de la edad de los 
niños. Dentro de estas observaciones, la practicante toma en cuenta en primer lugar; los momentos de exploración por grupos pequeños en diferentes espacios del aula que plantean las maestras para que los niños puedan crear diferentes juegos y desarrollar capacidades propias de la edad, en segundo lugar; el seguimiento de la rutina e instrucciones que brinda la maestra, y por último; el momento de patio observando la interacción entre pares, interacción docente - niño e interacción acompañante-niño (Anexo 3).

Luego de este proceso de observación, la practicante se reúne con la psicóloga a cargo para informar acerca de lo observado y poder realizar en conjunto una nueva observación sobre los puntos más resaltantes dentro de la rutina y si se presenta el caso de algún niño que debe ser observado con mayor minuciosidad.

Se acuerda, una segunda reunión con los padres dependiendo de cómo se presente el proceso de adaptación de cada niño para que se comente sobre los logros o las mejorías que se pueden realizar con el fin de que se consiga la autonomía e independencia del mismo. Asimismo, se presenta dos informes cualitativos a los padres (mayo y septiembre) elaborados por la docente y la practicante de psicología, supervisados por la psicóloga y coordinadora pedagógica, relatando el progreso en seis áreas determinadas y las recomendaciones propiamente según la edad y el desarrollo de cada niño (Anexo 4).

\section{2 Capacitación a las cuidadoras sobre el rol del acompañante en el proceso de} adaptación escolar.

Durante el proceso de adaptación, se presentan casos de niños con dificultad de desapego hacia sus acompañantes. Estos niños suelen necesitar un tiempo prolongado para lograr quedarse solos en este nuevo espacio, asumiendo mayor autonomía e independencia. Es por ello, que luego del segundo semestre escolar, el departamento de psicología realiza un plan de intervención enfocándose en la adaptación e independencia del niño dentro de la escuela. Se invita a los padres a inscribir a las cuidadoras y/o acompañantes de los niños. De esta manera, 
el departamento de psicología se contacta con ciertos padres vía correo electrónico proponiendo fechas y temas, brindándoles la opción de inscribir a las cuidadoras con las que trabajan a esta capacitación.

\section{2. 1 Taller: Capacitación para cuidadoras y acompañantes en la adaptación del}

\section{niño a la escuela}

- Objetivo general: ofrecer estrategias y recursos en su trabajo diario, en concordancia con el enfoque planteado en XYZ, con el fin de lograr la adaptación escolar de ambos niveles.

- Objetivos específicos:

- Primera sesión: informar sobre la filosofía de trabajo, que permita explicar los principios de XYZ y estrategias sobre una crianza respetuosa para el desarrollo integral del niño dirigido hacia cuidadoras.

- Segunda sesión: Realizar dinámicas de grupo (donde se enseñan propuestas de juego y relación con el otro) y presentar experiencias propias, para permitir el desarrollo de la teoría ya expuesta evidenciándola en el practica por medio de situaciones de los niños que tienen a cargo para poder trabajarlas en conjunto a manera de conversatorio.

- Tercera sesión: Unificar la primera y segunda sesión, que ayudará a evaluar la recepción del material teórico que se ha brindado buscando situaciones en las cuales las cuidadoras puedan plantear una solución basándose en la filosofía Reggio Emilia. 
- Procedimiento:

1. La psicóloga, la practicante de psicología y la docente se reúnen para comentar sobre los niños que aún cuentan con dificultades para poder mantenerse solos en la escuela sin acompañante.

2. La psicóloga y la practicante de psicología se reúnen con los padres de estos niños para brindarles estrategias y pautas para que sean comunicadas a las acompañantes.

3. Al no lograr el objetivo de que el niño se adapte, la practicante de psicología realiza un plan de intervención que consta de un taller de capacitación con tres sesiones para acompañantes/cuidadoras en el cual se acude a los padres para que las inscriban indicando día, hora y lugar del taller. (Anexo 5)

4. Cada taller cuenta con un objetivo específico que tiene como meta lograr el objetivo de adaptación del niño basándose en el enfoque que plantea XYZ.

5. Una parte del taller es realizado con la técnica de grupo de discusión (Bonilla Jimenez \& Escobar, 2017), el tema es planteado por el expositor de acuerdo con el objetivo de la sesión y las cuidadoras opinan, debaten y generan acuerdos o concesos sobre su rol.

6. Finalmente, las cuidadoras tienen un espacio en el cual pueden comentar dudas, consultas y recomendaciones.

\section{3 Charlas informativas para los padres}

\subsubsection{Taller: Generando un vínculo saludable con mi hijo}

- Objetivo general: Brindar talleres fomentar el vínculo entre padres e hijos.

- Objetivos específicos:

- Proponer estrategias para fortalecer el vínculo con el niño.

- Acompañar a los padres en el desarrollo integral. 
- Plantear estrategias para lograr una crianza respetuosa.

- $\quad$ Procedimiento:

1. La psicóloga y la practicante de psicología realizan una convocatoria vía correo electrónico indicando día, hora y lugar del taller para padres divido en primer nivel (1-2 años) y segundo nivel (3-5 años). El máximo de participantes es de 10 familias por taller. (Anexo 6)

2. Los talleres son desarrollados por los miembros del departamento psicopedagógico tomando en cuenta tres temas: El apego y vínculo, las etapas de desarrollo del niño (y sus logros en cada una), la crianza respetuosa.

3. Los padres se anotan en el taller de su preferencia y asisten dentro del día y horario indicado.

4. Dada la cantidad de miembros del taller es de 10 participantes, se realiza una breve introducción a los temas propuestos para luego iniciar una dinámica de grupo de discusión (Bonilla Jimenez \& Escobar, 2017) tomando en cuenta diferentes experiencias que permitan aplicar lo que se ha informado.

5. Se culmina el taller con una conclusión general sobre los temas que se han tratado y con sugerencias de los padres sobre nuevos temas que se puedan trabajar.

6. Finalmente se acepta también, la petición de algunos padres para tener una reunión individual con la psicóloga y practicante de psicología. 


\section{CAPÍTULO III: RESULTADOS DE LA INTERVENCIÓN}

En relación a las actividades realizadas se obtuvieron una serie de resultados. En lo que respecta al acompañamiento de los niños de 2-5 años en el proceso de adaptación escolar, se ha mostrado interés de los padres por asistir a las reuniones donde se brinda un informe cualitativo del niño basándose en una metodología de observación participante (Hernández Sampieri, Fernández Collado, \& Baptista Lucio, 2010) de acuerdo a lo establecido por la institución, evidenciando estrategias para su adaptación al nuevo espacio o brindando recomendaciones para que esto se logre. Sin embargo, a lo largo del primer semestre solo un grupo de 10 acompañantes de 42 lograron el objetivo de despedirse del niño para que permanezca de manera independiente en el espacio.

Al realizarse la capacitación para las cuidadoras, dentro de las 30 participantes que fueron inscritas en el taller para poder guiar de mejor manera el acompañamiento del niño dentro de este proceso escolar solo 18 de ellas obtuvieron el objetivo de que el niño pueda permanecer solo en el espacio de manera tranquila, relacionándose con sus pares y realizando las tareas propuestas de manera independiente. De manera que, 12 de ellas no lograron el objetivo para el segundo semestre y continuaron el acompañamiento hasta fin de año. Estos resultados podrían responder a que probablemente no se consideraron todos los aspectos de la teoría planteada anteriormente por Londoño (2013) en la que indica los componentes necesarios para propiciar un adecuado inicio en la etapa escolar: las características del desarrollo de los niños, reconocer los factores que influyen en la adaptación a nuevas situaciones, establecer relaciones con las familias, implementar estrategias que ayuden a los niños durante la transición. Dado que, para una buena adaptación y apego seguro tienen que darse estos cuatro, de manera conjunta generando una condición lista para que esto suceda. 
Con respecto al taller para padres, se ha encontrado ciertas limitaciones de los padres debido a que la mayoría de ellos trabaja y tiene responsabilidades (horarios de trabajo, hijos menores) que no le permite atender este tipo de necesidades que plantea la escuela en relación a la adaptación de sus hijos. Es así, que se cumple parcialmente la teoría que plantea (Geddes, 2010) en la que señala que las relaciones con los padres desempeñan un papel importante en el desarrollo de la capacidad infantil para relacionarse con el mundo exterior. Por lo que, la poca disponibilidad de tiempo genera en los niños la incapacidad de confiar en el cuidador principal y de sentirse seguros en un nuevo espacio.

Tanto el acompañamiento directamente con el niño y las familias, como los talleres implementados bridan un aporte desde el espacio psicológico al educativo centrándose en lo emocional, proponiendo experiencias relativamente novedosas que permiten aplicar conocimientos ya existentes pero validados de una manera diferente y en conjunto con la nueva filosofía educativa que presenta esta institución. Sin embargo, con un trabajo preventivo más minucioso se podría brindar un mayor aporte metodológico en las intervenciones realizadas para lograr el cumplimiento del objetivo trazado por el departamento psicopedagógico. 


\section{CONCLUSIONES}

Como se ha planteado en XYZ, tiene una filosofía educativa fundada en el modelo socio constructivista, modelo que es asumido con rigor tanto en el manejo conceptual como en el operativo. Por esta razón, en esta institución se requiere realizar un trabajo especial con los padres y con las cuidadoras para lograr que el proceso de adaptación a la escuela se desarrolle dentro de lo que la institución espera para este nivel y edades.

- El trabajo desarrollado se ha planeado siguiendo el modelo educativo de la institución, sin embargo, este podría contar con un registro más objetivo y detallado al momento de realizar las observaciones iniciales que determinan los avances de cada niño.

- 10 de 42 niños lograron adaptarse con facilidad, ante la evaluación realizada después de dos meses del ingreso. Los talleres sobre adaptación a la escuela se desarrollan en el segundo semestre tanto para padres como para cuidadoras. El resultado final es que 18 de los 32 niños logran adaptación a final de año. Concluyendo que es importante que la institución se anticipe a estos resultados, dándole un valor mayor al cambio de cronograma de actividades, implementando un tipo de intervención de manera preventiva al ingreso de los niños al año escolar (marzo).

- Los 12 niños que no logran adaptarse con este proceso de talleres continúan recibiendo el apoyo individual y la supervisión de la psicóloga con la practicante. Afinando la búsqueda de información sobre la dinámica familiar y el conocimiento de los entornos en los que se desarrolla el niño, para partir y enfocarse en ese motivo o motivos y poder abarcar el caso desde ahí, dándole estrategias puntuales. 


\section{RECOMENDACIONES}

- Replantear el cronograma de actividades con el fin de tener una visión que permita el trabajo más preventivo frente demandas que se han presentado con mayor frecuencia.

- A partir de las observaciones de corte naturalista de acuerdo a lo establecido por la institución, se podrían realizar fichas más sistemáticas que permitan una mirada objetiva del comportamiento y la conducta que presente el niño durante el proceso de adaptación de manera más específica.

- Desarrollar un aumento de las capacitaciones a la comunidad educativa con mayor reincidencia en las practicantes para que puedan involucrarse de mejor manera y puedan contar con una variedad de recursos para brindar acompañamiento a los niños y docentes.

- Reformular el contenido de los talleres, de manera que sean más vivenciales y se expongan casos particulares o de modelo, aplicando la teoría de manera cotidiana.

- Incrementar el número de psicólogos de acuerdo a la cantidad del alumnado, permitiendo un mayor estudio y análisis de los datos en relación a la información de cada uno y su adaptación. 


\section{REFERENCIAS}

Bautista Loza, N. E., \& Espinosa Anchaluisa, G. d. (2010). Investigación de la metodología utilizada en el período de adaptación de los niños y niñas de la sección pre-escolar de la unidad educativa experimentar "Colegio Militar Eloy Alfaro" de Quito. Sangolquí.

Bonilla Jimenez, F. I., \& Escobar, J. (2017). Grupo focales: una guía conceptual y metodológica. Cuadernos hispanoamericanos de psicología, 51-67.

Diloné, H., \& Pons, S. (2010). Acompañamiento pedagógico y profesionalización docente: sentido y perspectiva. Ciencia y Sociedad Republicana Dominicana, 521-541.

Geddes, H. (2010). El apego en el aula: relación entre las primeras experiencias infantiles, el bienestar emocional y el rendimiento escolar (Vol. 269). Barcelona: Graó.

Geenen, G., Corveleyn, J., \& Koroleff, P. (2014). Vinculos protectores: apego en padres e hijos en vulnerabilidad. En G. Geenen, Vinculos protectores: apego en padres e hijos en vulnerabilidad. Lima: Fondo editorial de la Pontificia Universidad Católica del Perú.

Gómez Martínez, A. (2018). Intervención de las familias en los centros de Educación Infantil. Soria: Universidad de Valladolid.

Hernández Sampieri, R., Fernández Collado, C., \& Baptista Lucio, P. (2010). Metodología de la investigación (Vol. 3). México: McGraw-Hill.

Londoño Palacio, M. M. (2013). La adaptación escolar y social en niñas de preescolar: un análisis a partir de las relaciones de aceptación y rechazo en el grupo de pares. Bogotá: Universidad de la Sabana.

Moneta C, M. (2014). Apego y pérdida: redescubriendo a John Bowlby. Revista Chilena de Pediatría, 265-268.

Navia Antezana , C., \& Villareal Delgado, A. (2013). Asesoría e identidad profesional en educación preescolar. Educere, 299-307.

Sainz De Vicuña, P. (2009). Educar en el aula de 2 años. Una Propuesta metodológica para el aula de niños y niñas de 2 años. Revista Electrónica Interuniversitaria de Formación del Profesorado, 35-55.

Valencia Chávez, N. (3 de Noviembre de 2017). Ver, Escuchar y Sentir Metodología Reggio: Estudio en primera infancia. Ver, Escuchar, Sentir Metodología Reggio: Estudio en primera infancia. Obtenido de iesmonturiol.net: www.iesmonturiol.net/web/html/estudis/recerca/tdr_nicolasvalencia.pdf

Vélez Vasco, I. C., \& Vergara M, C. E. (2015). Acompañamiento de los docentes en proceso de adaptación escolar de niñas y niños de Preescolar en el Colegio Tercer Milenio. Caldas-Antoquia: Corporación Universitaria Lasallista. 
Woods Winnicott, D. (1986). Las necesidades del niño y el papel de la madre en las primeras etapas. En El niño y el mundo externo. Buenos aires: Horme.

Yábar Tito, C. S., \& Bronzoni Zlatar, L. V. (2018). Desarrollo de la autonomía en niños de 18 a 24 meses de edad según la filosofía de Reggio Emilia aplicada en el Nido La Casa Amarilla. Lima: Pontificia Universidad Católica Del Perú. 


\section{APÉNDICES}




\section{APÉNDICE 1: Modelo de entrevista con padres}

Esquema de preguntas para entrevista / primera reunión con padres

1. Datos personales (nombre del niño, fecha de nacimiento, alguna complicación durante el embarazo, alergias, como está conformada la familia, quien está a cargo del niño, oficio de los padres, etc.)

2. Rutina del niño/a

3. ¿Cómo sería un día ideal para (nombre de niño/ niña)?

4. ¿ Cómo sería un día ideal para ti con (nombre de niño/niña)?

5. Experiencias pre escolares previa

6. Acompañante para el proceso de adaptación 


\title{
APÉNDICE 2: Cartilla del acompañante
}

\author{
¿Cómo ayudar al niño en sus primeros días en el nido? \\ RECOMENDACIONES PARA EL ACOMPAÑANTE \\ CREEMOS EN EL GRAN POTENCIAL DE LOS NIÑOS, POR ESO NO LES ENSEÑAMOS \\ AQUELLO QUE PUEDEN APRENDER POR SÍ MISMOS.
}

1.- Permite que la niña/el niño participe y explore por iniciativa propia, sin que se sienta presionado a ello. No lo empujes para que se despegue de ti ni para que toque los juguetes. Si no lo hace y necesita estar cerca de ti acompáñalo en sus intereses. Lo hará solo cuando esté listo para ello.

2.- Los primeros días acompaña a tu hijo en las exploraciones, juega con él/ella; esto le dará seguridad y lo hará sentirse confiado en el espacio. Una vez que se haya dado el traslado de confianza hacia las maestras trae siempre una actividad personal propia de adultos y discreta, para que realices durante las horas que estés acompañando al niño en el nido (revista, tejido, sudoku, agenda). Si no tienes, las profesoras te pueden prestar una revista o lecturas sobre desarrollo infantil.

3.- Mientras estés en el nido, mantén el celular apagado y evita ingerir alimentos. Si las profesoras te han pedido permanecer sentada en un lugar, permanece allí porque tu niño podría ir a buscarte en cualquier momento y necesitamos encontrarte ahí. Si no te encuentra, su confianza se deteriora. Si necesitas moverte por alguna razón avísale; por ejemplo, "Alonso necesito ir al baño, voy un ratito y vuelvo a mi silla".

4.- Permite que el niño ingrese y salga del nido caminando por sí mismo, no lo cargues en tus brazos. Mejor ofrécele tu mano, y nunca lo jales del brazo ni de la muñeca.

5.- La puntualidad en la hora de llegada y salida es fundamental para que el niño se sienta seguro.

Cuando te vayas y dejes a tu hijo en el nido:

Para ayudar a que se quede tranquilo, es fundamental que te despidas de él. Cuando lo hagas, la despedida debe ser tranquila, clara y de una sola vez: "Marcela, me voy a trabajar, a las 12 viene la abuela por ti. En la tarde regreso a la casa para bañarte y estar juntas".

\section{El niño confiará si percibe que tú también confías en las maestras para hacerse cargo. Muchas gracias por tu colaboración.}




\section{APÉNDICE 3: Modelo de ficha de observación}

Ficha de registro de conducta

Niño ( ) Niña ( ) Nombre:

Fecha de registro:

Edad:

Tiempo

de

observación:

Conductas observadas

Seguimiento de la rutina ( seguimiento de
instrucciones y adaptación a las reglas
de grupo)

Momentos de exploración por grupos

pequeños (participación en las

actividades, colaborador $\mathrm{u}$ iniciativa)

Momento de juego libre en el patio (

interacción entre pares, con el docente y

con el acompañante)

\section{Registro descriptivo}




\title{
APÉNDICE 4: Formato de informe escrito
}

XYZ

\author{
INFORME PEDAGÓGICO
}

\section{Nombre del niñóa}

\section{Primer nivel 1-2 años}

1. Cuidados (lonchera, higiene, cambios de pañal y de ropa) (vínculo, lenguaje, autonomía, sentido de competencia, relación con su propio cuerpo, hábitos, modales, responsabilidades, matemática activa, colaboración)

2. Pensamiento lógico matemático/literacidad - gráfica: a partir de las nociones y aprendizajes, cómo las trabaja el niño en las áreas de investigación/cuidados u otros momentos.

3. Trabajo por áreas de investigación (aprendizajes: competencias, habilidades, conceptos, estrategias de colaboración y de alta demanda cognitiva, actitudes frente al trabajo, autonomía,

enriquecimiento del lenguaje): cómo trabaja el niño en grupo pequeño, el proceso de elaboración y reparación de teorías, préstamo de ideas, trabajo con lenguajes, dibujo esquemático.

4. Contextos de investigación/proyectos (alta demanda cognitiva, metacognición, trabajo en equipo, habilidades motrices, pensamiento creativo, procesos de construcción de teorías,

liderazgo).

5. Patio (habilidades motrices, trabajo en equipo, creación de juegos y dinámicas en colaboración, cuidado del patio, retos, iniciativa).

6. Cuentos (capacidad de escucha y atención, participación, pensamiento creativo, vocabulario, enriquecimiento del lenguaje, lectura de imágenes). 


\section{Segundo nivel 3-4 años}

1. Pensamiento lógico matemático/literacidad - gráfica: a partir de las nociones y aprendizajes, cómo las trabaja el niño en las áreas de investigación/cuidados u otros momentos.

2. Trabajo en áreas de investigación (aprendizajes: competencias, habilidades, conceptos, estrategias de colaboración y de alta demanda cognitiva, actitudes frente al trabajo, autonomía, enriquecimiento del lenguaje): cómo trabaja el niño en grupo pequeño, el proceso de elaboración y reparación de teoría, prestamos de ideas, trabajo con lenguajes, dibujo esquemático.

3. Trabajo en contextos de aprendizaje-investigaciones (alta demanda cognitiva, metacognición, trabajo en equipo, habilidades motrices, pensamiento creativo, procesos de construcción de teorías, liderazgo).

4. Reunión de reflexión (escucha, hábitos, expresión verbal, metacognición, participación, organización de las ideas).

5. Patio (habilidades motrices, trabajo en equipo, creación de juegos y dinámicas en colaboración, cuidado del patio, retos, iniciativa).

6. Cuentos (capacidad de escucha y atención, participación, pensamiento creativo, vocabulario, enriquecimiento del lenguaje, lectura de imágenes). 


\section{APÉNDICE 5: Formato de inscripción para capacitación de cuidadoras}

\section{Inscripciones - Capacitación de cuidadoras o acompañantes}

Lugar: $X Y Z$

Horario: 9:00-12:00pm

Fecha: Primera, segunda y tercera semana de septiembre (Lunes, Miércoles y Viernes)

Temas propuestos
Primera sesión: Principios de XYZ y estrategias sobre una crianza respetuosa.
Segunda sesión: Dinámicas de grupo y exposición de casos
Tercera sesión: Evaluar la recepción del contenido
1.
2.
3.
4.
5.
6.
7.
8.
10.




\section{APÉNDICE 6: Formato de inscripción de taller para padres}

\section{Inscripciones - Talleres para padres}

Lugar: XYZ

Horario: 7:00-9:00pm

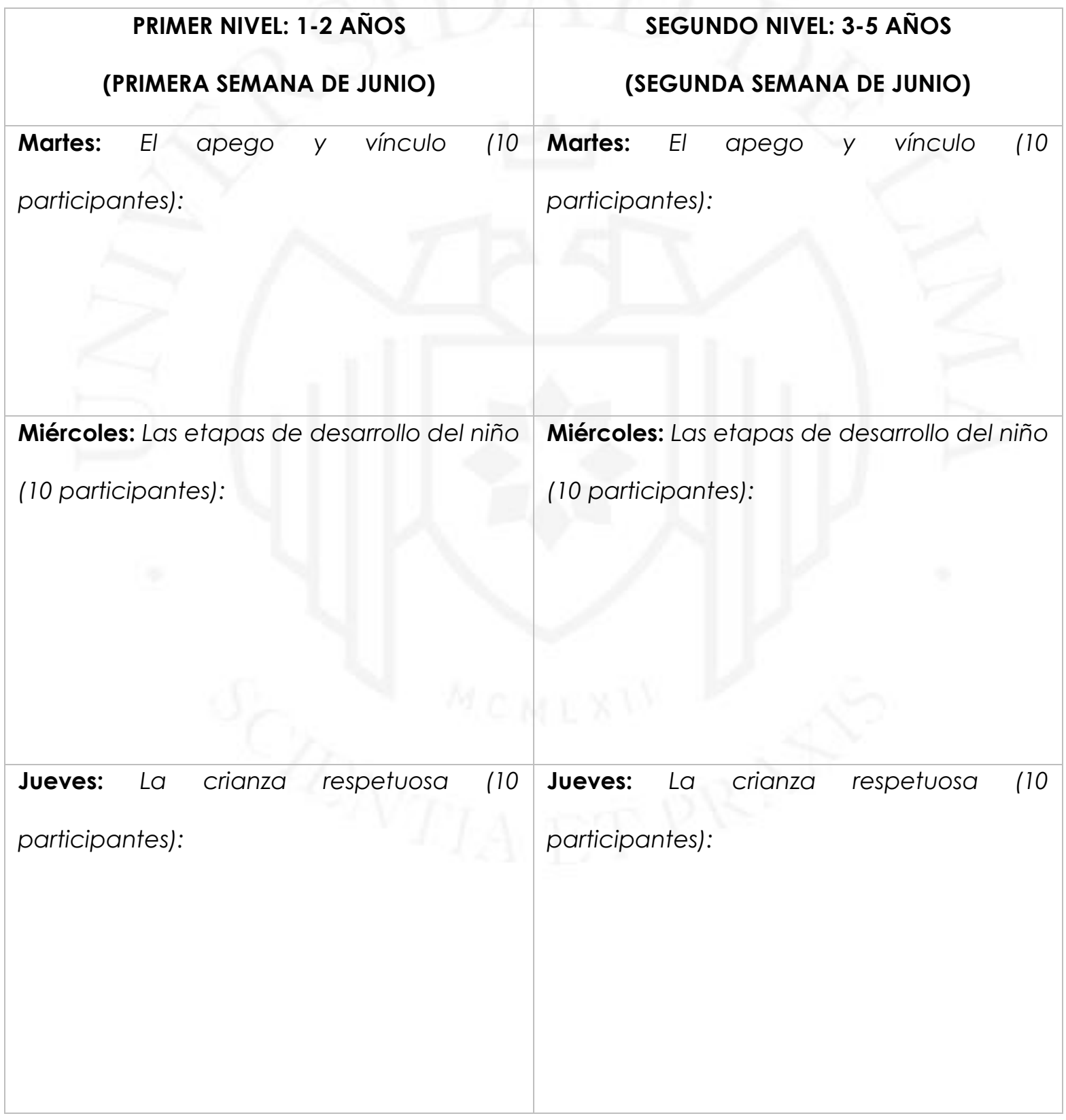

